Modern Physics Letters A

(C) World Scientific Publishing Company

\title{
LESSONS FROM ALL LOGS SUMMATION IN YUKAWA THEORIES
}

\author{
I. F. GINZBURG \\ Sobolev Institute of Mathematics SB RAS, pr. ac. Koptyug, 4, Novosibirsk 630090, Russia \\ ginzburg@math.nsc.ru \\ Novosibirk State University, ul. Pirogova, 2, Novosibirsk 630090, Russia
}

Received 19.10.2009

\begin{abstract}
Some features of old results in the total summation of all logarithmic contributions of all diagrams in Yukawa theory are presented. We discuss some lessons from this picture for the description of Pomeron, odderon, etc. in QCD.
\end{abstract}

Keywords: Summation of asymptotics; Yukawa theories; gauge theory

$12.38 \mathrm{Cy}, 11.55 \mathrm{y}, 12.39 \mathrm{St}$

\section{Introduction}

The recipes for the asymptotics of the Feynman diagrams in dependence on its topology at arbitrary Lagrangian were obtained in 60 -th (see Refs. 112 and references therein). The reduction of problem to the special case of diffractive processes $A B \rightarrow C D$ at

$$
\left.s=\left(p_{A}+p_{B}\right)^{2} \approx-u=\left(p_{A}-p_{D}\right)^{2} \gg|t|=\mid\left(p_{A}-p_{C}\right)^{2}\right) \mid, m_{i}^{2}
$$

in the Yukawa theory (with pseudoscalar or scalar charged bosons $\phi_{i}$ )

$$
L=4 \pi g \bar{\psi} \gamma^{5} \tau^{i} \psi \phi_{i}+(4 \pi)^{2} \lambda\left(\phi_{i}^{2}\right)^{2}
$$

allows us to obtain very simple recipes suitable for more ambitious problems 3 .

Based on these results we summarized all logarithmic contributions of all diagrams for scattering amplitude, assuming finite charge renormalization ${ }^{4}$. The final results were published in reviews $\frac{5}{5}$. Variations of results for running coupling constants were almost evident based on approach from 6 .

We used the Mellin transform of amplitude defined separately for positive and negative signature

$$
\begin{gathered}
f^{ \pm}(s, t) \equiv \frac{f(s, t) \pm f(u, t)}{2} \\
f^{ \pm}(s, t)=\frac{i}{2} \int_{i-\infty}^{+\infty} d j\left(\frac{u-s}{2}\right)^{j} \xi^{ \pm}(j) F^{ \pm}(j, t) ; \quad \xi^{ \pm}(j)=\frac{1 \pm e^{i \pi j}}{2 \Gamma(j+1) \sin \pi j}
\end{gathered}
$$


with inverse transformation defined in the standard manner. The asymptotical behavior in $s$ is determined by the most right singularity of $F(j, t)$ in the complex $j=$ plane. This point $j=j_{0}$ is the same for each diagram for given $A B \rightarrow C D$ process in the fixed theory (Yukawa, $\phi^{3}$, gauge theory).

For the contributions of separate diagrams we used Feynman type $\alpha$ representation described e.g. in Ref. ${ }^{7}$ and developed in Ref. ${ }^{[1}$. We use normalization of amplitudes in which $d \sigma \propto|f|^{2} / s$ without additional dimensional factors for all processes with both bosons and fermions.

With suitable normalization for the amplitudes with fermions in the Yukawa theory these leading singularities of separate diagrams are formed from poles at $j=0$ as

$$
F_{\text {diagr }}^{Y}=\sum a_{k} j^{-k}
$$

It corresponds $f_{\text {diagr }}(s)=\sum \tilde{a}_{k} \log ^{k} s$. The poles at $j=-1$ describe higher twist corrections.

In the gauge theory like QED or QCD contributions of separate diagrams for flavor singlet $t$-channel contain multiple poles in the points $j=n>0$ with integer $n$ with gauge dependent coefficients. However, contributions of different diagrams strongly cancel each other so that the gauge invariant contribution of each order of perturbation theory is formed from poles at $j=1$ as

$$
F_{p e r t, n}^{G}=\sum b_{k}(j-1)^{-k}
$$

It corresponds $f_{\text {pert }, n}(s)=s \sum \tilde{b}_{k} \log ^{k} s$. The poles at $j=0$ describe higher twist corrections.

In the $\phi^{3}$ theory we have $F_{\text {diagr }}^{\phi^{3}}=\sum c_{k}(j+1)^{-k}$. It corresponds $f_{\text {diagr }}(s)=$ $\sum \tilde{c}_{k} \log ^{k} / s$.

The mentioned cancellation of contributions of separate diagrams don't allow us to apply methods developed for Yukawa theory in the gauge theory. Nevertheless, we think that some features of results obtained in Yukawa theory can have relation to the description of complete picture in the gauge theory.

\section{The amplitude with positive signature}

We discuss first the processes with vacuum number exchange in $t$-channel $(A \rightarrow C)$ and with positive signature.

\subsection{LLA results}

The simplest picture appears in scalar theory $(g=0)$. In this case the LL contributions of perturbation theory are given by simple ladder diagrams. In the leading $\operatorname{logs}$ approximation (LLA) amplitude was found in Refs. $\frac{8}{6}$ as solution of quadratic 
equation

$$
\begin{gathered}
v_{L L}=F_{L L A, g=0}, \quad v_{L L}^{2}-j v_{L L}+r_{L L}=0, \quad r_{L L}=240 \lambda^{2} \Rightarrow \\
\Rightarrow v_{L L}=\frac{1}{2}\left(j+\sqrt{j^{2}-4 r}\right) .
\end{gathered}
$$

The LLA amplitude has fixed square-root branch point in the $j$-plane at $j=2 \sqrt{r}$, i.e. $f(s, t) \propto s^{2 \sqrt{r}}(\ln s)^{-3 / 2}$.

Next, we consider fermions with Yukawa interaction. In this case one must consider different two-particle states in the $t$-channel. That are two-boson states $(M)$ and fermion-antifermion $(f \bar{f})$ states with projection to vector $(V)$. (Other $f \bar{f}$ states don't contribute to the vacuum number exchange amplitude.) In this case LLA result (6) takes place with simple variation. Both $v_{L L}$ and $r_{L L}$ become matrices $2 \times 2$ with components $(M, V)$. In particular, in the case of flavor $S U(2)$ symmetry

$$
r=\left(\begin{array}{cc}
240 \lambda^{2} & \sqrt{6} g^{2} \\
\sqrt{6} g^{2} & 3 g^{2}
\end{array}\right)
$$

\subsection{Complete amplitude}

The detail description of asymptotics of all diagram allows to summarize all logarithmic contributions of all diagrams, i.e. all pole contributions (4).

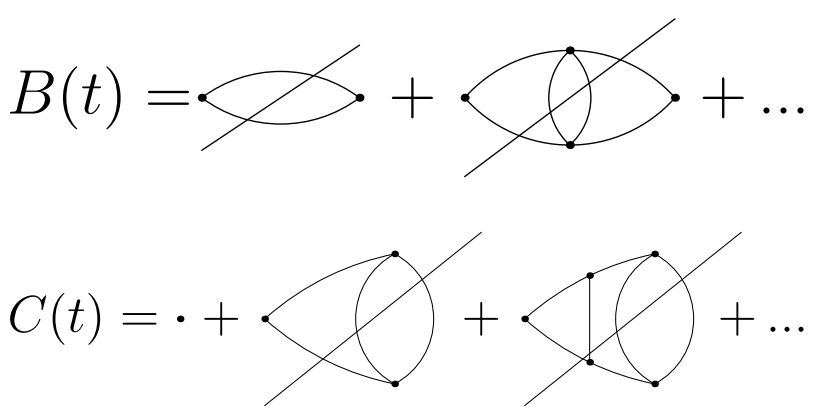

Fig. 1. Diagrammatic representation for $B(t)$ and $C(t)$.

The results has form of $3 \times 3$ matrix representation with components $(M, V, T)$ :

$$
F(j, t)=C_{j}^{T}(t)\left(\frac{1}{v^{-1}(j)-B_{j}(t)}\right) C_{j}(t) .
$$

Here matrices $B(t)$ and $C(t)$ are described by diagrammatic series of Figs. 1 where each term is specifically regularized (oblique lines in pictures), it has no singularities at $j>-1$, their $t$-dependence is most important fact. Matrix $v(j)$ accumulates all poles at $j=0$. It can be also presented in the diagrammatic form where each term is independent on $t$ and particle masses. In the case of finite charge renormalization $v(j)$ has fixed square-root branch point in the $j$-plane. It is obtained 
from quadratic equation system of form (6), where $r$ become $3 \times 3$ matrix with components $(M, V, T)$; the LLA for this matrix is given by the Eq. (7) (with $T$ components equal to 0 ). For the weak coupling case the NLL terms of $v(j)$ are easily calculated and fixed branch point position is shifted.

In the case of running coupling constant $v(j)$ has fixed singularity in the $j$-plane, as before. To describe it, following $[6$ one must analyze some subsidiary integral equation for $v(j)$.

It is easy to understand how this result will be changed if vacuum expectation values of some products of field operators are non-zero (vacuum condensate). In this case only equation for $B$ and $C$ are changed - by adding of diagrams with insertion of condensate blobs within diagrams shown in Figs. 1.

Finally, this result gives representation of scattering amplitude, containing both fixed points in $j$-plane and Regge-poles (zeroes of denominator (8) ). In our example we cannot say even what of the contributions is dominant, the LLA type fixed singularity in j-plane or Regge pole. Note that this representation presents clear and unambiguous factorization - all small distant contributions are contained in $\mathrm{v}(\mathrm{j})$ while large distance contributions are contained in $\mathrm{B}(\mathrm{t})$ and $\mathrm{C}(\mathrm{t})$.

We don't discuss in more details specific features of result in the Yukawa theory.

\subsection{Lessons}

(1) Complete amplitude has generally form, strongly different from that of LLA. In our example we cannot speak even what contribution is dominant in real amplitude, LLA similar fixed singularity in $j$-plane or Regge pole.

(2) Improvements of LLA, similar to NLLA, NNLLA are often misleading. In our example they give corrected form for $v(j)$ and some general coefficient dependent on $t$ instead of correct quite different behavior.

\section{Types of leading singularities of diagrams}

The studies of asymptotics of diagrams allow to distinguish two types of singularities of diagrams in $j$-plane (see 112 ) in the case of finite masses of all participating particles.

- Small distance (end-point) singularities. In the $\alpha$-representation they originate from the region where for some lines $\alpha_{i} \rightarrow 0$. These singularities appear in both planar and non-planar diagrams.

- Pinch singularities. In the $\alpha$-representation they originate from the region with generally non-zero $\alpha_{i}$ due to some compensation between coefficients. These singularities include effects of both small and large distance effects. They appear in non-planar diagrams only. In each theory the pinch singularities in diagrams are responsible for the poles in $j$-plane in the same point $j=j_{0}$ as for small distance singularities.

For the considered class of theories we prove ${ }^{3}$ the theorem, describing responsibility of different types of singularities for asymptotics of amplitude for summation 
of poles $\left(j-j_{0}\right)^{-n}$.

- At even $j_{0}$ (in particular, for $j_{0}=0$ )

- the amplitude with positive signature is described by small distance singularities only,

- the pinch contributions together with small distance are responsible for the amplitude with negative signature.

- At odd $j_{0}$ (in particular, for $j_{0}= \pm 1$ )

- the amplitude with negative signature is described by only small distance singularities,

- the pinch contributions together with small distance are responsible for the amplitude with positive signature.

The summation of asymptotics with pinch singularities is very complicated problem. Only the LL results were published here. For the $\phi^{3}$ theory (with some preliminary estimates for theories with higher $j_{0}$ ) it was done in Ref. 2 . These results were

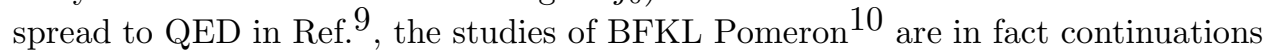
of this activity for $\mathrm{pQCD}$.

\section{Odderon/ Pomeron}

Let us remind two points.

1. Pomeron is amplitude with positive signature, odderon is amplitude with negative signature.

2. In the theory $\phi^{3}$ we have $j_{0}=-1$; in the Yukawa theory we have $j_{0}=0$; in the gauge theory (QED, QCD) we have $j_{0}=1$.

Therefore:

In the Yukawa theory $j_{0}=0$, and the asymptotics of Pomeron amplitude is determined by only small distance singularities of diagrams. This very fact allowed us to summarize all logarithmic asymptotics of all diagrams in the simple form (8). The odderon amplitude, which is determined by both small distance and pinch singularities, was not calculated till now even in LLA.

In the $\phi^{3}$ theory $j_{0}=-1$, and the asymptotics of Pomeron amplitude is determined by both small distance and pinch singularities of diagrams. It was found in LL as solution of some integral equation 2 . Vice versa, the asymptotics of odderon amplitude has much simpler structure, it is determined by only small distance singularities of diagrams. The total summation of all logarithmic contributions of all diagrams was presented in Ref. $[5$.

In the gauge theory (QED and QCD) $j_{0}=1$, and the asymptotics of Pomeron amplitude is determined by both small distance and pinch singularities of diagrams. It is very complex problem. The LL results were obtained in Ref.10. Recent NLL results are note very clear for me due to reasons, discussed in sect. 2.3. Vice versa, the asymptotics of odderon amplitude has much simpler structure, it is determined by only small distance singularities of diagrams. The corresponding direct analysis, starting from diagrams, was not done till now. The representation 
$6 \quad$ I.F. Ginzburg

$$
\text { odderon }=\text { colored Pomeron }+ \text { gluon }
$$

looks for us misleading.

\section{Acknowledgment}

I discuss here mainly the results obtained in our collaboration with Anatoly Efremov and our pupils. I recall this collaboration as the most pleasant in my life. I am very thankful organizers of ECT meeting "Recent Advances in Perturbative QCD and Hadronic Physics" at July 20-25, 2009 for invitation. This paper was supported by grants RFBR 08-02-00334-a and NSh-1027.2008.2.

\section{References}

1. A.V. Efremov. Preprints JINR R-1242, 1305 (1963), E-2125 (1965); G. Tiktopoulos. Phys. Rev. 131 480, 2373 (19630; A.V. Efremov, O.I. Zavjalov. Proc. Dubna Conf. (1965), p.380

2. R.J. Eden, P.V. Landshoff, D.J. Olive, J.C. Polkinghorne. The analytic S-matrix. Cambridge (1966)

3. I.F. Ginzburg, A.V. Efremov, V.G. Serbo. Sov. Yad. Fiz. 9 (1969) 451; I.F. Ginzburg, V.G. Serbo. Sov. Yad. Fiz. 9 (1969) 868; V.M. Budnev, I.F. Ginzburg. Sov. J. Theor. Math. Phys. 3 (1970) 171

4. V.M. Budnev, I.F. Ginzburg, V.G. Serbo. Sov. J. Theor. Math. Phys. 6 (1971) 55; I.F. Ginzburg. Lett. Nuovo Cim. 7 (1973) 155.

5. A.V. Efremov, I.F. Ginzburg. Fortschr. Physik 22 (1974) 575-609; Proc. Int. Conf. on Mathem. problems of QFT and Quantum statistics, Moscow, 1972; Trudy Mathem. Inst. of USSR Acad. of Sc. (Moscow), 136 (1975) 39; A.V.Efremov, I.F. Ginzburg, V.G. Serbo. Proc. Int. Sem. on anal. properties of scattering amplitude, June 1969, Serpuchov (1969).

6. I.F. Ginzburg, V.V. Serebryakov. Sov. Yad. Fiz. 3 (1966) 164; I.F. Ginzburg, V.G. Vasjev. Sov. Yad. Fiz. 5 (1967) 669.

7. N.N. Bogolyubov, D.V. Shirkov. Introduction in the theory of quantized fields N-Y, Interscience (1959)

8. R.F. Sawyer Phys. Rev. 131 (1966) 1384

9. V.N. Gribov, L.N. Lipatov, G.V. Frolov. Sov. J. Nucl. Phys. 12 (1971) 543

10. See e.g. E.A. Kuraev, L.N. Lipatov, V.S. Fadin. Sov. Phys. JETP 45 (1977) 199 


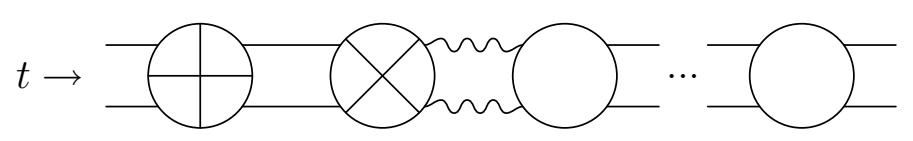

